\title{
Expression and Mutational Analyses of the Human MAD2LI Gene in Breast Cancer Cells
}

\author{
Melanie J. Percy,' Kenute A. Myrie, ${ }^{2}$ Christopher K. Neeley,' James N. Azim,' Stephen P. Ethier, ${ }^{3}$ and \\ Elizabeth M. Petty ${ }^{1,2 *}$ \\ 'Department of Internal Medicine, University of Michigan Medical Center, Ann Arbor, Michigan \\ ${ }^{2}$ Department of Human Genetics, University of Michigan Medical Center, Ann Arbor, Michigan \\ ${ }^{3}$ Department of Radiation Oncology, University of Michigan Comprehensive Cancer Center, Ann Arbor, Michigan
}

Breast cancer is a heterogeneous disorder in which most tumors display some degree of aneuploidy, especially those at later stages of the disease. Aneuploidy and associated chromosome instability may be important in the progression of mammary tumorigenesis. Aneuploidy is prevented during normal cell division in part through regulation of a mitotic spindle checkpoint where mitotic arrest prevents segregation of misaligned chromosomes into daughter cells at anaphase. Mitotic arrest genes, including the MAD family, which was originally characterized in yeast, help regulate normal function of the mitotic spindle checkpoint. Decreased expression of the human gene MAD2LI was previously reported in a breast cancer cell line exhibiting chromosome instability and aneuploidy. To explore further the potential role of MAD2LI in breast cancer, we analyzed MAD2LI gene expression in 13 minimally to grossly aneuploid human breast cancer cell lines and found significant differences of expression in three lines. Sequence analysis of MAD2LI cDNA in these as well as nine additional aneuploid breast cancer and five immortalized normal human mammary epithelial cell lines revealed one heterozygous frameshift ( 572 del $A)$ mutation in a cancer cell line that demonstrated a high level of transcript expression. In addition, two 3'UTR sequence variants were noted in breast cancer cell lines. The 572 del A mutation creates a truncated MAD2 protein product. Further functional studies in primary breast tumors are therefore warranted to determine the potential role MAD2LI may play in breast cancer.

() 2000 Wiley-Liss, Inc.

Breast cancer continues to be a significant cause of morbidity and mortality in the Western world. It is widely hypothesized that breast cancer arises from the clonal accumulation of mutations in genes regulating normal cellular growth, akin to the elegant multistep paradigm first recognized in colorectal cancer (Fearon, 1997). The multistep pathway(s) in breast cancer remains poorly understood, however, especially for the common heterogeneous sporadic mammary neoplasms in which no predisposing germline mutations exist. Examination of mutation rates in somatic cells implies that the clonal acquisition of sufficient mutations to promote multistep tumorigenesis is improbable during a human lifetime (Loeb, 1991; Orr-Weaver and Weinberg, 1998). Additional molecular mechanisms may be required to drive the cell toward a malignant phenotype. In some solid tumors, especially some colon cancers, malignant progression is accelerated when the cells' genomes are rendered unstable by alterations in genes that have defective DNA mismatch repair ability, allowing the rapid accumulation of mutations to drive tumorigenesis (Lengauer et al., 1998). Most breast cancers do not harbor mismatch repair defects to account for increased genomic instability; however, a majority exhibit some degree of aneuploidy, especially at later stages of the disease (Shackney et al., 1995). The significance of observable aneuploidy in breast cancer is not well established, but correlates with both disease aggressiveness (Tsuda et al., 1998) and chromosomal instability (CIN) (Duesberg et al., 1998; Lengauer et al., 1998). Molecular defects resulting in CIN and aneuploidy, therefore, may contribute to the progression of mammary tumorigenesis.

Normally, a mitotic spindle checkpoint monitors proper microtubule attachment to chromosomes prior to progression through mitosis to yield euploid daughter cells through regulation of the anaphase promoting complex (APC) (Lengauer et al., 1998). Yeast genes important in the spindle checkpoint include members of the $B U B$ (budding uninhibited by benomyl) and $M A D$ (mitotic arrestdeficient) families. Early evidence suggests that alterations of the human homologs of these genes

Supported by: NIH; Contract Grant number: KO8CA66613-01; Wendy Will Case Cancer Fund (to EMP); ROI (to EMP); Grant number: CA72877-02

*Correspondence to: Elizabeth M. Petty, Department of Internal Medicine, Division of Medical Genetics, 4301 MSRB III, University of Michigan, 1150 West Medical Center Drive, Ann Arbor, MI 48109. E-mail: epetty@umich.edu

Received 11 May 1999; Accepted 6 June 2000 
may in fact be associated with human malignancy. Reduced expression of the human MAD2L1 transcript was previously reported in T47D, a human breast cancer cell line that demonstrated a defective mitotic arrest response (Li and Benezra, 1996). However, further studies exploring the potential role of MAD2L1 in breast cancer are lacking. MAD2L1, located on 4q27, encodes a 205-aminoacid protein that is associated with the kinetochore of unattached chromosomes and inhibits APC, but is absent when chromosomes are correctly aligned on the metaphase plate (Chen et al., 1996). MAD2 protein exists as a monomer or as a tetramer, with the latter state likely being the active form that inhibits APC (Fang et al., 1998a, 1998b). Oligomerization of MAD2 or a conformation associated with the MAD2 tetrameric state may transduce the mitotic checkpoint signal (Wolf and Jackson, 1998). Mutations in BUB1, a human homolog to another yeast mitotic arrest gene, demonstrated functional importance in human colorectal cancers exhibiting chromosome instability. Specifically, dominant negative mutant $B U B 1$ alleles in colorectal tumor cell lines promoted CIN and were associated with abnormal mitotic checkpoint responses (Cahill et al., 1998). Lee et al. (1999) recently demonstrated that mutations in Bub1 and Mad3L mitotic spindle checkpoint genes can potentiate growth and cellular transformation in a study of Brca2-deficient murine cells. It would appear that defects in Brca2 and mitotic spindle checkpoint genes could work together to overcome checkpoint controls in the cell cycle, thereby promoting cell division, resulting in aneuploidy, and driving tumorigenesis. These findings suggest that similar alterations in other mitotic arrest genes may play roles in breast cancer by abolishing the normal mitotic checkpoint control mechanism. Loss of normal control would allow mammary epithelial cells to divide even when chromosomes are not correctly attached to the spindle, giving rise to aneuploidy and chromosomal instability that may accelerate tumor progression.

Because decreased expression of MAD2L1 was previously described in one breast cancer cell line (Li and Benezra, 1996), we elected to explore further involvement of MAD2L1 in breast cancer. We analyzed MAD2L1 transcript expression in 13 breast cancer cell lines to help determine the frequency of altered expression of MAD2L1 in breast cancer. Given that many of these well-characterized cell lines are markedly aneuploid, we hypothesized that they would be likely to have the highest probability of harboring alterations in MAD2L1 if, indeed, alterations of this gene were important in aneuploidy and breast cancer.

Ten SUM breast cancer cell lines, 12 American Type Culture Collection breast cancer cell lines, and 5 human papilloma virus (HPV) immortalized normal breast cell lines were initially available for study. SUM breast cancer and normal breast cell lines were developed at the University of Michigan Comprehensive Cancer Center by Stephen P. Ethier and are well characterized with regard to their cytogenetic phenotype (Ethier et al., 1993, 1996; Garcia et al., 1997; Sartor et al., 1997; Flanagan et al., 1998; Forozan et al., 1998; Ignatoski and Ethier, 1998). These cell lines were cultured in Ham's F-12 medium, either serum-free or with 5\% FBS, in the presence of Fungizone and gentamycin. In addition, insulin, hydrocortisone and epidermal growth factor or choleratoxin were included in the medium (see http://p53.cancer.med.edu/clines/ clines/elab.html). The breast cancer cell line CAL51 was kindly provided by Dr. J. Gioanni, Nice, France (Gioanni et al., 1990) and grown in Dulbecco's modified Eagle medium (DMEM) supplemented with $10 \%$ fetal bovine serum (FBS). The remaining breast cancer cell lines were maintained according to the American Type Culture Collection instructions.

Total RNA was isolated from all cell lines at approximately $80 \%$ confluency using Trizol reagent according to the manufacturer's instructions (GibcoBRL, Grand Island, NY). For Northern blot analysis, $10 \mu \mathrm{g}$ of total RNA from 13 breast cell lines was fractionated on $1.25 \%$ agarose gel and transferred to Hybond $\mathrm{N}^{+}$(Amersham Life Science, Piscataway, $\mathrm{NJ})$ in $10 \times \mathrm{SSC}$. A 1.3-kb MAD2L1 full-length RTPCR product (5-TGTCGGCGGAGTGGAAGC-3; 5-ACTTTATT'TCGTCACTT'TCA-3) and a 407-bp GAPDH RT-PCR product (5-GGGAGCGAAAAGGGTCATCA-3; 5-TTTCTAGACGGCAGGTCAGGT-3) were labeled by random priming with $\left[\alpha^{32} \mathrm{P}\right]$ dCTP. Hybridization was performed at $60^{\circ} \mathrm{C}$ overnight in Church buffer (1-mM EDTA, 0.5-M $\mathrm{NaHPO}_{4}, 7 \%$ SDS). Filters were washed at $60^{\circ} \mathrm{C}$ in $2 \times \mathrm{SSC}, 0.1 \%$ SDS, and exposed to Kodak XAR-5 film.

By Northern blot analyses, all MAD2L1 transcripts were of the anticipated $1.4-\mathrm{kb}$ size, and no aberrant transcripts were detected. MAD2L1 expression levels were visually assessed as compared to ethidium bromide-stained gels for loading and also standardized against glucose-3-phosphate-dehydrogenase $(G A P D H)$ gene expression levels by calculating the ratio of MAD2L1 to GAPDH for all cell lines as quantified by densitometry (data not 
Lane $\quad \begin{array}{llllllllllllll}1 & 2 & 3 & 4 & 5 & 6 & 7 & 8 & 9 & 10 & 11 & 12 & 13\end{array}$

A

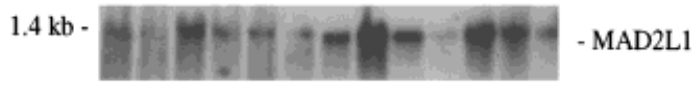

B

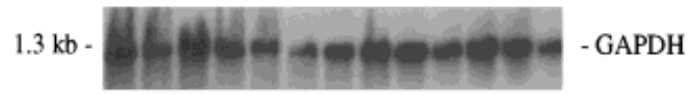

Figure I. Expression analyses of MAD2LI in breast cancer cell lines. A: ${ }^{32-}{ }^{32-l a b e l e d ~ f u l l-l e n g t h ~ R T-P C R ~ M A D 2 L I ~ p r o b e ~ d e t e c t s ~ a ~ I .4-k b ~}$ band for each of the breast cancer cell lines: MDA-MB-36I (lane I), MDA-MB-435s (lane 2), BT-20 (lane 3), BT-474 (lane 4), BT-549 (lane 5), DU4475 (lane 6), MCF7 (lane 7), CAL5 I (lane 8), SK-BR-3 (lane 9), Hs578T (lane I0), SUMI49PT (lane II), SUMI59PT (lane I2), and SUMI3I5MO2 (lane I3). B: GAPDH RT-PCR probe illustrates RNA loading.

shown, Alpha Innotech IS-1000 Digital Imaging System, San Leandro, CA). Densitometry was used to confirm our visual observations in all cases. The ratio of MAD2L1 expression to GAPDH expression in an immortalized normal mammary epithelia line with a functionally normal mitotic checkpoint in response to chemical disruption of the mitotic spindle microtubules by nocodazole, 5-24 HPV, was determined to be 0.8 (data not shown). This $M A D 2 L 1$-to- $G A P D H$ transcript expression ratio was nearly identical to the ratios observed for the cancer cell lines MCF7 and SK-BR-3 (lanes 7 and 9, Fig. 1). The levels of MAD2L1 expression in the BT-20 and SUM1315MO2 lines (lanes 3 and 13, Fig. 1) were similar but slightly less with ratios of $M A D 2 L 1$ to $G A P D H$ closer to 0.6. In the MDA-MB361, MDA-MB-435s, BT-474, BT-549, DU4475, and Hs578T lines (lanes 1, 2, 4, 5, 6, and 10, Fig. 1), MAD2L1 expression appeared comparatively low by visual analysis and MAD2L1-to-GAPDH expression ratios as determined by densitometry were calculated to be $<0.5$. As shown, MAD2L1 expression was most dramatically reduced in lines MDAMB-435 and Hs578T where the MAD2L1-to$G A P D H$ ratios were $<0.1$. Compared to other lines, CAL51, SUM149PT, and SUM159P'T demonstrated increased MAD2L1 expression as determined by both visual observation and densitometry analysis (lanes 8, 11, 12, Fig. 1). Most significantly, CAL51 demonstrated significantly increased expression with an MAD2L1-to-GAPDH ratio > 3.0. The physiologic importance of variable expression in these cell lines is difficult to assess because appropriate matched control samples were not available for analysis. It is possible that variable expression could be due to differences in cell cycle states. Other factors such as the rate of cell growth when the cells were harvested for RNA isolation could also contribute to the variable levels of tran- script expression, but all attempts were made to minimize this possibility by harvesting the cells at $80 \%$ confluence. Southern blot analysis did not reveal any structural changes that could explain the variability in MAD2L1 expression levels in these breast cancer cell lines (data not shown). Given the variable expression and the difficulty inherent in interpreting transcript expression analysis without matched controls, we elected to analyze these and additional breast cancer lines further for mutations.

We conducted a mutational analysis of the MAD2L1 gene in 22 breast cancer cell lines and 5 HPV immortalized normal breast epithelial cell lines by direct sequencing of RT-PCR amplified MAD2L1 gene products to determine whether there were alterations in the coding region. RTPCR was performed with $5 \mu \mathrm{g}$ of total RNA using the SuperScript Preamplification System (GibcoBRL) and oligo dT primers. A 1.3-kb fragment encompassing the conceptual translation start site of the MAD2L1 open reading frame and the $3^{\prime}$ untranslated region (UTR) was amplified using primers 5-TGTCGGCGGAGTGGAAGC-3 (F1, Fig. 2) and 5-ACTTTATTTCGTCACTTTCA-3 (R1, Fig. 2) (Krishnan et al., 1998). This avoided the amplification of a pseudogene homologous to the $5^{\prime}$ region of MAD2L1 located on chromosome 14 (Krishnan et al., 1998). PCR products were purified using the High Pure PCR Product Purification Kit (Boehringer Mannheim, Indianapolis, IN) and were quantified fluorometrically. Sequencing was performed using a Thermosequence Cycle Sequencing Kit (Amersham Life Science) with the following primers: 5-GCGCGTGCTTTTGTTTGT-3 (S1), 5-CCAGAGAAAAGTCTCAGAAAGC-3 (S2), and 5-GGCAGAAATGTCACCGTAGC-3 (S3) (Fig. 2). Reaction products were separated by electrophoresis on $6 \%$ polyacrylamide urea gels in $1 \times$ glycerol tolerant buffer (USB). Gels were dried and exposed to Kodak XAR-5 film.

MAD2L1 cDNA was generated from all of the breast cancer cell lines, and one major band of expected size $1.3 \mathrm{~kb}$ was amplified for each cell line. A deletion of $\mathrm{A}$ at nucleotide 572 within the coding region (Genbank accession number U31278) was identified in the CAL51 breast cancer cell line leading to a premature stop codon (Fig. 3). This deletion was confirmed from two independent cDNA samples. Because the mutant and normal sequences were obtained from CAL51 cDNA and the primers used to amplify the MAD2L1 gene from cDNA do not amplify genomic DNA, the expressed transcript contained both wild-type and mutant alleles. The loss of nucleotide 572 causes a 


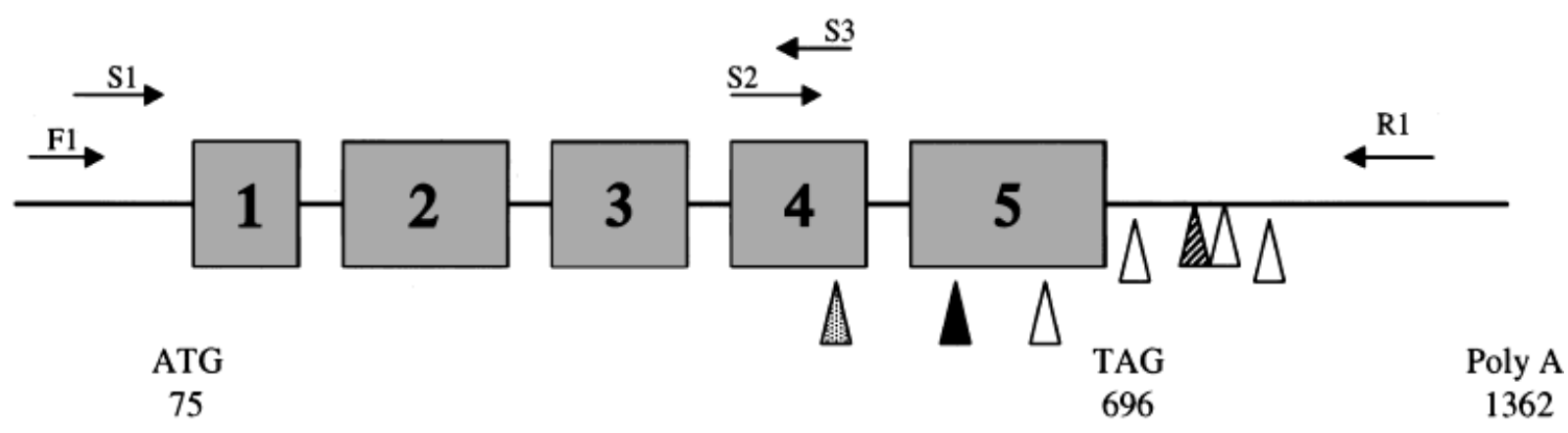

Figure 2. MAD2LI gene structure. Shaded boxes indicate the five exons. Primers used for PCR (FI, RI) and sequencing (SI, S2, S3) are shown by horizontal arrows. Black triangle indicates 572 del A in CAL5I breast cancer cell line; open triangles indicate sequence variants

frameshift mutation, resulting in the substitutions of the subsequent 27 amino acids after residue 167 . In addition, there is loss of the terminal 13 amino acids due to the premature stop at codon 193. This has significant consequences for the MAD2 protein because it has been reported that the 10 terminal amino acids of MAD2 contain the region necessary for binding to CDC20 and oligomerization (Luo et al., 2000). It has also been demonstrated in yeast that this C-terminal tail is involved in Mad1 and Mad2 binding, which is required for checkpoint function (Fang et al., 1998a). No nucleotide changes were detected in the first 500 nucleotides of the coding region. This sequence has been reported to contain conserved surface residues that form two distinct patches of a central $\beta$-sheet in the MAD2 protein (Luo et al., 2000).

Two sequence variants were also detected in the $3^{\prime} \mathrm{UTR}$ of the MAD2L1 gene in breast lines. Eight of 27 breast lines exhibited a heterozygous G-to-A transition at nucleotide 751 (Table 1). MDA-MB157 carried a T-to-C transition at nucleotide 837 (Table 1). To examine further the prevalence of the sequence variants in other cancer lines, sequence analysis of MAD2L1 was performed on cDNA from 24 nonbreast cancer cell lines (ATCC). The 751 (G-to-A) transition was observed in one allele of seven cell lines (total 15 of 51 breast and nonbreast cancer cell lines), suggesting that this sequence variant is most likely to be a polymorphism. No other cell lines carried the 837 ('T-to-C) transition. In addition, four single sequence variants were detected in MAD2L1 transcript in the nonbreast cancer cell lines. Three of these cell lines, U-87MG, U-373MG, and MDA-H2774, exhibited an A-to-G transition at the wobble nucleotide 503 for proline 143. An A-to-G transition at nucleotide 642 changed isoleucine to valine at found in breast cancer and nonbreast cancer cell lines. Striped triangle denotes a frequently found sequence variant, and dotted triangle denotes a sequence variant in a nonbreast cancer cell line (U-87MG) that results in an isoleucine to valine substitution. Figure not drawn to scale.

codon 190 in cell line U-87MG (Fig. 3). Because both amino acids have similar characteristics, this substitution may represent a benign polymorphism. At nucleotide positions 709 and 780 in the 3'UTR, A-to-G substitutions were detected in $\mathrm{U}-87 \mathrm{MG}$ and $\mathrm{OV}-1063$.

To investigate whether the MAD2L1 mutation (572 del A) detected in the breast cancer cell line CAL51 generated a truncated MAD2 protein product as predicted, we carried out in vitro transcription/translation of MAD2. The 1.3-kb RT-PCR products from CAL51 were TA subcloned into PCR II-TOPO vector (Invitrogen, Carlsbad, CA). Sequencing confirmed the presence or absence of the mutation. Wild-type and mutant inserts of $M A D 2 L 1$ were directionally cloned into pcDNA3.1 expression vector (Gibco-BRL) using Bam $\mathrm{H} 1$ and $X b a 1$ sites. Correct sequence orientation was confirmed by sequencing. In vitro transcription/translation of the cloned mutant and wild-type copies of MAD2L1 was performed using the TNT T7 Quick Coupled Transcription/Translation System (Promega, Madison, WI) according to the manufacturer's instructions. Reaction products were electrophoresed on a $15 \%$ SDS-PAGE gel, transferred to Hybond-P nitrocellulose (Amersham Life Science) by semidry transfer, immunoblotted with primary goat MAD2 IgG polyclonal $\left(1: 1,000, \mathrm{NH}_{3}\right.$ terminus) antibody and secondary antigoat IgG HRPconjugated antibody $(1: 8,000)$ (Santa Cruz Biotechnology, Santa Cruz, CA), and visualized by chemiluminescence using Supersignal West Pico ECL kit (Pierce, Rockford, IL). As predicted, the mutant allele resulted in a truncated protein approximately $22.2 \mathrm{kD}$ in size (Fig. 4), a difference of $1.3 \mathrm{kD}$ from the predicted wild-type MAD2 protein of $23.5 \mathrm{kD}$ (Li and Benezra, 1996). Analyses by Western blot of $150 \mu \mathrm{g}$ of CAL51 cell lysate did not 

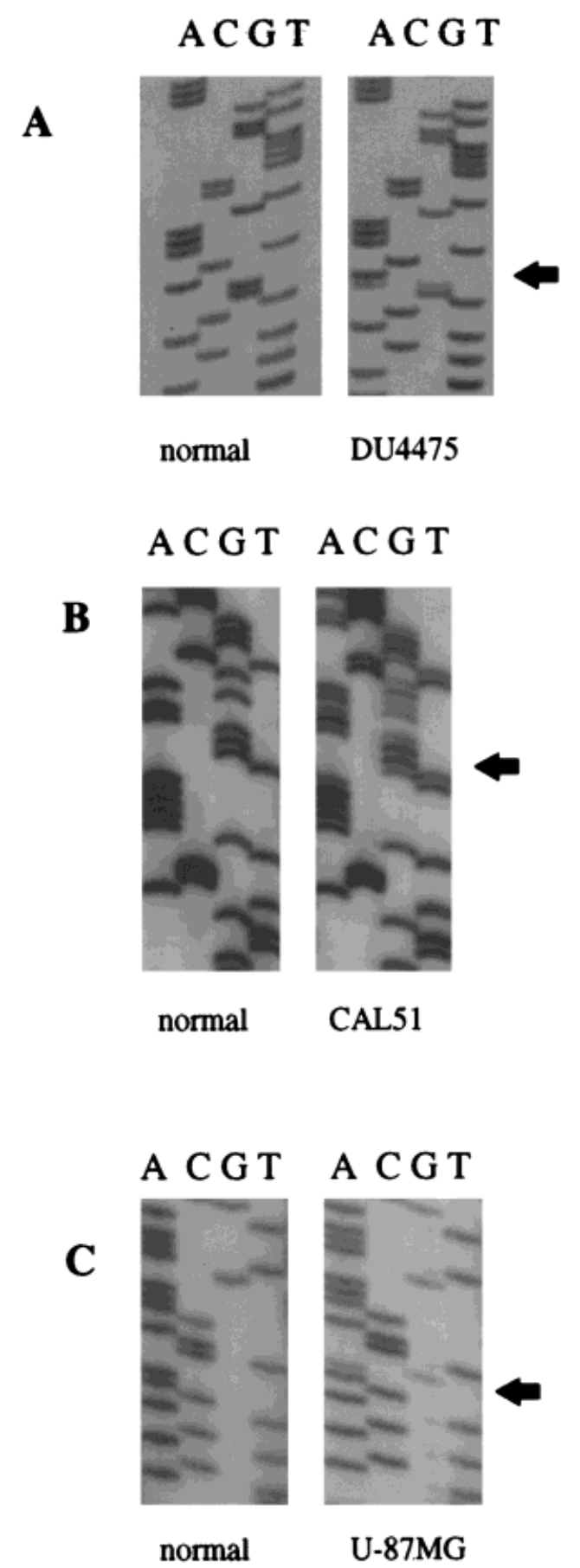

Figure 3. MAD2LI sequence variants. A: Breast cancer cell line DU4475 has a change at nucleotide 75I (G-to-A) also detected in eight breast cancer cell lines and one nonbreast cancer cell line. B: Deletion of $A$ at nucleotide position 572 (572 del A) in CAL5 I breast cancer cell line causes a frameshift and a premature stop codon; thus, apparent slippage of sequence of one allele is noted (mutant sequence TTGTACCTGAAAAATGGGAAGAGTC . . . ; underlined base is deleted from one allele). $\bar{C}$ : A transition at nucleotide 642 (A-to-G) detected in U-87 MG nonbreast cell line. Arrows points to bands corresponding to base changes; a normal sequence is included for comparison. reveal an endogenous truncated protein (data not shown), suggesting that the truncated protein may be unstable or that it is expressed at very low levels undetectable by this analysis.

MAD2 forms a ternary complex with CDC20 and APC. Binding of MAD2 inhibits activation of the APC by CDC20, causing arrest at metaphase during the cell cycle (Fang et al., 1998a). Inhibition of the APC ensures fidelity of sister chromatid separation by controlling defects in the kinetochore attachment to the spindle and correct initiation of anaphase. In vivo evidence suggests that MAD2 exists as a monomer and a tetramer (Fang et al., 1998a). Both forms bind to CDC20, but in vitro studies demonstrate that only the tetramer inhibits CDC20 activation of the APG (Fang et al., 1998a). The 572 del A of MAD2 in the CAL51 breast cancer cell line is predicted to cause a change in $20 \%$ of the carboxy end of the protein. Although the function of this mutation has not yet been characterized, given that MAD2 functions as a tetramer, one abnormal allele could have a dominant negative effect. Loss of function of MAD2 through a dominant negative mutation could promote aneuploidy by abrogating the mitotic spindle checkpoint. Truncated MAD2 would most likely undergo a conformational change that alters binding to CDC20 and therefore regulation of the APC. Similarly, the truncated protein may bind to CDC20, but is unable to prevent degradation of anaphase inhibitor and activation of the CDC20APC complex because it cannot oligomerize to form the active protein. Alternatively, the truncated protein may be unstable and expressed at relatively low levels. In CAL51 cells, both mutant and wild-type MAD2L1 alleles are expressed, as suggested by Northern blot analysis and RT-PCR. Northern blot data suggested an increased level of MAD2L1 transcript expression, potentially reflecting compensatory overexpression due to lack of normal function. Given this, it is currently unclear how the heterozygous 572 del A mutation would effect the mitotic checkpoint and/or cellular proliferation in CAL51 cells. Studies of this mutation through in vitro and in vivo model systems will be needed to determine if the mutation can in fact function as a dominant negative alteration. Presently, it is also unclear how the expression of the $M A D 2 L 1$ gene is regulated during the cell cycle. Overexpression of MAD2 in yeast causes mitotic arrests at metaphase (He et al., 1997) and would suggest an exertion of spindle checkpoint control. Therefore, increased expression may be a mechanism that compensates for loss of normal function 
TABLE I. Mutational Analysis of MAD2LI in Immortalized Normal and Transformed Breast Cancer Cell Lines

\begin{tabular}{|c|c|c|c|c|}
\hline Cell line & $\begin{array}{l}\text { Lanes as on } \\
\text { Northern blot }\end{array}$ & Ploidy status & Base change & Mutation status \\
\hline MCF- $10^{\mathrm{a}}$ & & Diploid & No change & \\
\hline 4-I2 HPV & & Diploid & $75 \mathrm{I}(\mathrm{G} \rightarrow \mathrm{A})^{\mathrm{b}}$ & Heterozygous \\
\hline 5-24 HPV & & Diploid & No change & \\
\hline II-6 HPV & & Diploid & No change & \\
\hline$I I-2 I_{H P V}{ }^{a}$ & & Diploid & $75 \mathrm{I}(\mathrm{G} \rightarrow \mathrm{A})^{\mathrm{b}}$ & Heterozygous \\
\hline BT-20 & Lane 3 & Hyperdiploid & No change & \\
\hline BT-474 & Lane 4 & Aneuploid & No change & \\
\hline BT-549 & Lane 5 & Aneuploid & No change & \\
\hline CAL5I & Lane 8 & Diploid to tetraploid & $572(\operatorname{del~A})^{c}$ & Heterozygous \\
\hline DU4475 & Lane 6 & Aneuploid & $75 \mathrm{I}(\mathrm{G} \rightarrow \mathrm{A})^{\mathrm{b}}$ & Heterozygous \\
\hline Hs $578 \mathrm{~T}$ & Lane 10 & Aneuploid & $75 \mathrm{I}(\mathrm{G} \rightarrow \mathrm{A})^{\mathrm{b}}$ & Heterozygous \\
\hline MCF-7 & Lane 7 & Hypertriploid to hypertetraploid & No change & \\
\hline MDA-MB-I 57 & & Hypotriploid & $837(\mathrm{~T} \rightarrow \mathrm{C})^{\mathrm{b}}$ & Homozygous \\
\hline MDA-MB-23I & & Aneuploid & No change & \\
\hline MDA-MB-36I & Lane I & Hyperdiploid to hypodiploid & No change & \\
\hline MDA-MB-435s & Lane 2 & Aneuploid & No change & \\
\hline SK-BR-3 & Lane 9 & Hypertriploid to hypotetraploid & No change & \\
\hline SUM-44PE & & Aneuploid & No change & \\
\hline SUM-52PE & & Aneuploid & No change & \\
\hline SUM-I02PT & & Aneuploid & $75 \mathrm{I}(\mathrm{G} \rightarrow \mathrm{A})^{\mathrm{b}}$ & Heterozygous \\
\hline SUM-I49PT & Lane II & Aneuploid & No change & \\
\hline SUM-I59PT & Lane 12 & Near diploid & $75 \mathrm{I}(\mathrm{G} \rightarrow \mathrm{A})^{\mathrm{b}}$ & Heterozygous \\
\hline SUM-I85PE & & Aneuploid & No change & \\
\hline SUM-I90PT & & & $75 \mathrm{I}(\mathrm{G} \rightarrow \mathrm{A})^{\mathrm{b}}$ & Homozygous \\
\hline SUM-225CWN & & & No change & \\
\hline SUM-229PE & & & No change & \\
\hline SUM-I3I5MO2 & Lane 13 & Aneuploid & $75 \mathrm{I}(\mathrm{G} \rightarrow \mathrm{A})^{\mathrm{b}}$ & Homozygous \\
\hline
\end{tabular}

Locations of nucleotide changes are denoted as follows: ${ }^{a}$ immortalized normal breast epithelial cell lines; ${ }^{b}$ sequence variants in the $3^{\prime}$ UTR; ' $d e l e t i o n$ in coding region.

of the mutant protein in CAL51 cells. CAL51 cells are minimally aneuploid, near-diploid in many cells, with a high proportion of tetraploid cells. Interestingly, analysis of gastrointestinal preneoplastic lesions suggests that tetraploidization may be one of the earliest consequences of asymmetric chromosome segregation secondary to dysregulation of genes that normally exert active checks on chromosome stability (Giaretti, 1997). Further analysis of CAL51 cell response to microtubule inhibitors is warranted for investigation of this hypothesis.

Whereas variable levels of transcript expression may be of no physiologic consequence, the apparent increased level of MAD2L1 transcript expression in a breast cancer line with a frameshift mutation suggests that they may reflect physiologic regulation. The detection of other breast cancer cell lines demonstrating variable levels of transcript expression despite no coding region mutations suggests that there could be mutations in transacting factors or cis-sequence elements that regulate MAD2L1 expression levels. Such factors could involve the $3^{\prime} \mathrm{UTR}$, where most of the sequence variants were located, but this remains to be deter- mined. There are no clear correlations between ploidy status, transcript expression levels, and $3^{\prime}$ UTR variants that can be made from this limited study. Interestingly, however, two of the breast cancer cell lines (MCF7 and SK-BR-3) that demonstrated MAD2L1 transcript expression levels closest to that seen in an HPV immortalized normal mammary epithelial cell line (5-24-HPV) did not have any identifiable sequence alterations. Of the eight breast cancer cell lines with comparatively less observable MAD2L1 expression, three of the lines do exhibit 3'UTR sequence alterations. Of the two lines suggesting increased MAD2L1 expression that lack an exon mutation, one line, SUM159PT, has the same 751 (G-to-A) transition in the $3^{\prime} \mathrm{UTR}$ region that is seen in two lines with decreased MAD2L1 expression. Factors such as posttranslation modification and different cell cycle states may also affect MAD2L1 expression. It is also possible that RT-PCR-based mutation analysis may not always detect an existing mutation in breast cancer lines with decreased MAD2L1 expression, especially if the mutant allele is not expressed at high enough levels. However, the fact that we did identify a se- 


\section{MUT WT marker}

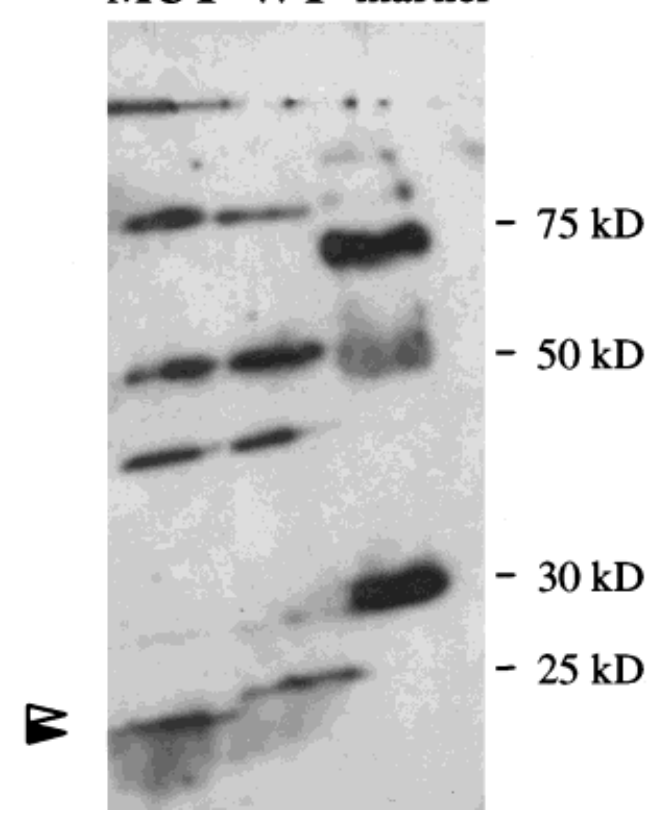

Figure 4. Immunoblot of MAD2 in vitro transcription/translation products from CAL5I breast cancer line. Open arrow indicates wildtype MAD2 of predicted size, $23.5 \mathrm{kD}$. Closed arrow indicates the predicted truncated MAD2 protein (mutant), approximately $22.2 \mathrm{kD}$ in size. Del 572 A results in a frameshift, amino acid substitution, and loss of terminal 13 amino acids due to premature stop at codon 193.

quence variant in one line with a very low level of MAD2L1 expression, Hs 578T, suggests that we were able to detect at least some sequence alterations in lines with low MAD2L1 expression using an RTPCR-based mutation screening approach.

Our studies to date suggest that additional analysis of the role of MAD2L1 in chromosome instability and mammary tumor progression is warranted. The role of MAD2L1 in breast cancer remains to be determined by further analysis in primary tumors as well as functional assays in vitro and in vivo.

\section{ACKNOWLEDGMENTS}

We thank E. Fearon for providing cancer cell lines and for critical reading of the article, F. Spencer for advice concerning RT-PCR amplification of MAD2L1, and J. Gioanni for the CAL51 cell line. We also thank C. Ammerman for technical assistance with cultivation of the SUM breast cancer cell lines and I. Maine for providing technical assistance with the protein truncation test. We thank L. Kalikin, E. Erson, A. Loeb, D. Robins, D. Burke, and T. Glover for helpful comments.

\section{REFERENCES}

Cahill DP, Lengauer C, Yu J, Riggins GJ, Willson JK, Markowitz SD, Kinzler KW, Vogelstein B. 1998. Mutations of mitotic checkpoint genes in human cancers. Nature 392:300-303.
Chen RH, Waters JC, Salmon ED, Murray AW. 1996. Association of spindle assembly checkpoint component XMAD2 with unattached kinetochores. Science 274:242-246.

Duesberg P, Rausch C, Rasnick D, Hehlmann, R. 1998. Genetic instability of cancer cells is proportional to their degree of aneuploidy. Proc Natl Acad Sci USA 95:13692-13697.

Ethier SP, Mahacek ML, Gullick WJ, Frank TJ, Weber BL. 1993. Differential isolation of normal luminal mammary epithelial cells and breast cancer cells from primary and metastatic sites using selective media. Cancer Res 53:627-635.

Ethier SP, Kokeny KE, Ridings JE, Dilts CA. 1996. erbB family receptor expression and growth regulation in a newly isolated human breast cancer cell line. Cancer Res 56:899-907.

Fang G, Yu H, Kirschner MW. 1998a. The checkpoint protein MAD2 and the mitotic regulator CDC20 form a ternary complex with the anaphase-promoting complex to control anaphase initiation. Genes Dev 12:1871-1883.

Fang G, Yu H, Kirschner MW. 1998b. Direct binding of CDC20 protein family members activates the anaphase-promoting complex in mitosis and G1. Mol Cell 2:163-171.

Fearon E. 1997. Human cancer syndromes: clues to the origin and nature of cancer. Science 278:1043-1050.

Flanagan L, Van Weelden K, Ammerman C, Ethier SP, Welsh J. 1999. SUM-159PT cells: A novel estrogen independent human breast cancer model system. Breast Cancer Res Treat 58:193-204.

Forozan F, Veldman R, Ammerman CA, Parsa NZ, Kallioniemi A, Kallioniemi O, Ethier SP. 1998. Molecular cytogenetic analysis of 11 new breast cancer lines. Br J Cancer 81:1328-1334.

Garcia R, Yu CL, Hudnall A, Catlett R, Nelson KL, Smithgall T, Fujita DJ, Ethier SP, Jove R. 1997. Constitutive activation of Stat 3 in fibroblasts transformed by diverse oncoproteins and in breast carcinoma cells. Cell Growth Diff 8:1267-1276.

Giaretti W. 1997. Aneuploidy mechanisms in human colorectal preneoplastic lesions and Barrett's esophagus: is there a role for K-ras and p53 mutations? Anal Cell Path 15:99-117.

Gioanni J, Le Francois D, Zanghellini E, Mazeau C, Ettore F, Lambert J, Schneider M, Dutrillaux B. 1990. Establishment and characterisation of a new tumorigenic cell line with a normal karyotype derived from a human breast adenocarcinoma. $\mathrm{Br} \mathrm{J}$ Cancer 62:8-13.

He X, Patterson TE, Sazer S. 1997. The Schizosaccharomyces pombe spindle checkpoint protein mad2p blocks anaphase and genetically interacts with the anaphase-promoting complex. Proc Natl Acad Sci USA 94:7965-7970.

Ignatoski KM, Ethier SP. 1998. Constitutive activation of pp125fak in newly isolated human breast cancer cell lines. Breast Canc Res Treat 54:173-182.

Krishnan R, Goodman B, Jin DY, Jeang KT, Collins C, Stetten G, Spencer F. 1998. Map location and gene structure of the Homo sapiens mitotic arrest deficient 2 (MAD2L1) gene at $4 \mathrm{q} 27$. Genomics 49:475-478.

Lee H, Trainer AH, Friedman LS, Thistlethwaite FC, Evans MJ, Ponder BAJ, Venkitaraman AR. 1999. Mitotic checkpoint inactivation fosters transformation in cells lacking the breast cancer susceptibility gene, Brca2. Mol Cell 4:1-10.

Lengauer C, Kinzler KW, Vogelstein B. 1998. Genetic instabilities in human cancers. Nature 396:643-649.

Li Y, Benezra R. 1996. Identification of a human mitotic checkpoint gene: hsMAD2. Science 274:246-248.

Loeb LA. 1991. Mutator phenotype may be required for multistage carcinogenesis. Cancer Res 51:3075-3079.

Luo X, Fang G, Coldiron M, Lin Y, Yu H, Kirschner MW, Wagner G. 2000. Structure of the Mad2 spindle assembly check-point protein and its interaction with Cdc20. Nat Struct Biol 7:224-229.

Orr-Weaver TL, Weinberg RA. 1998. A checkpoint on the road to cancer. Nature 392:223-224.

Sartor CI, Dziubinski ML, Yu CL, Jove R, Ethier SP. 1997. Role of epidermal growth factor receptor and STAT-3 activation in autonomous proliferation of SUM-102PT human breast cancer cells. Cancer Res 57:978-987.

Shackney SE, Singh SG, Yakulis R, Smith CA, Pollice AA, Petruolo S, Waggoner A, Hartsock RJ. 1995. Aneuploidy in breast cancer: a fluorescence in situ hybridization study. Cytometry 22:282-291.

Tsuda H, Sakamaki C, Tsugane S, Fukutomi T, Hirohashi S. 1998. A prospective study of the significance of gene and chromosome alterations as prognostic indicators of breast cancer patients with lymph node metastases. Breast Canc Res Treat 48:21-32.

Wolf DA, Jackson PK. 1998. Cell cycle: oiling the gears of anaphase. Curr Bio 8:R636-R639. 